\title{
Analyzing the Critical Sources of Dimensional Variability during the Lifecycle of a Steel Framed Modular Construction Project
}

\author{
Christopher RAUSCH ${ }^{1 *}$, Lichen ZHANG ${ }^{2}$, Jeffrey $\mathrm{WEST}^{3}$, Carl HAAS ${ }^{3}$ \\ ${ }^{1}$ MASc candidate, Department of Civil Engineering, University of Waterloo \\ ${ }^{2}$ Undergraduate research assistant, Department of Civil Engineering, University of Waterloo \\ ${ }^{3}$ Professor, Department of Civil Engineering, University of Waterloo \\ "Corresponding author's e-mail: ctrausch@uwaterloo.ca
}

\begin{abstract}
This paper presents a case study which analyzes critical sources of dimensional variability in a modular steel framed construction project. If not managed properly, dimensional variability can lead to conflicts during alignment and interfacing of components and modules. The management of dimensional variability can be expressed in distinct categories based on its impact on structural safety, constructability, aesthetics and functionality. This case study explores the use of a laser scanner and a total station in order to quantify critical sources of dimensional variability. The results of the case study show that during different project stages (i.e., fabrication, assembly, transportation, handling and erection) that often one of the distinct impact categories governs in terms of the management of dimensional variability. As such, this paper demonstrates how critical sources of dimensional variability can be identified so that they can be properly managed.
\end{abstract}

\section{KEYWORDS}

Dimensional variability; Tolerances; Modular construction; Steel frame assemblies; Prefabrication

\section{INTRODUCTION}

Variability associated with dimensions and geometry of construction components is inevitable due to process capabilities and geometric changes experienced throughout the construction lifecycle (i.e., fabrication, transportation and erection) of a project. This dimensional variability (which also encompasses geometric variability) can accumulate in large prefabricated assemblies resulting in conflicts and discrepancies between as-designed and as-built states. While the probability of a worst-case compounding effect of variability for an assembly is quite low in most cases (Silva 2012), structures comprised of heavy, rigid cross-sectional elements with very high relative stiffness can be very challenging to bring into correct alignment on site as the result of dimensional variability (Muir 2015). This is especially true for large scale modular steel frame structures. As the number of modules and lack of adjustability at connections increases, so does the difficulty of alignment. As such, it is necessary to understand sources of variability in order 
to control them or tailor the design to accommodate them. This paper presents a case study of a steel framed modular construction project where critical sources of variability are identified and subsequently quantified. This paper investigates more than just isolated construction component and assembly deviation values. For this purpose, there are numerous helpful guides which can be used to determine expected 'normal' dimensional variations (e.g., Handbook of Construction Tolerances). Rather, this paper looks at the compounding effect of dimensional variability in a modular structure. The approach taken for quantification of deviations is through the use of 3D imaging (laser scanning) and accurate 3D coordinate measuring (total station). The use of deviation analysis as presented by Rausch et al. (2016) is used as a means of quantifying deviations obtained from data collected. Critical sources are identified and discussed so that designers can make better decisions about how to manage dimensional variability in similar projects.

\section{BACKGROUND}

Variations of construction assemblies can be significantly larger than expected tolerances since there is often minimal understanding of how tolerances should be considered during design, controlled during construction, and enforced during inspection (Birkeland and Westhoff 1971; Milberg and Tommelein 2005). Consequently, the need to identify and quantify sources of dimensional variability becomes important as the construction industry shifts from traditional construction methods towards modular and prefabricated construction methods. Studies have found that variations in construction can be attributed to the lack of tolerances and explicit instructions specified in design details (Malisch and Suprenant 2005; Posey et al. 2007). As such, complications can be inherent in design details, indicating the need for a greater understanding of why tolerances are needed and how to specify adequate tolerance values.

The interface of different materials (e.g., precast concrete members bearing on structural steel members) creates conflicting tolerances and can result in components that do not fit (Malisch and Suprenant 2005). To avoid excessive rework, tolerances in each material group need to be consistent to develop overall assembly tolerances. However, relaxing a tolerance may cause a product to not operate properly while tightening a tolerance increases the construction cost. As such, optimization is required to provide satisfactory performance at reasonable cost. This is why identification of critical sources of dimensional variability is important for determining their governing impact in different construction stages. In practice, designers rarely make explicit provision for variation when planning details of construction. They often rely on standards to specify tolerances and on builders to "do the cutting and remedial work required to make the several parts of the work come together properly" (Posey et al. 2007).

There is often a lack of understanding of when stricter tolerances than those specified by codes or standards is needed. For instance, most construction literature which covers information about tolerances, discusses general classes or categories for tolerances (AISC 2010a; ACI 2002; Silva 2012; AISC 2010b). Among these categories however, is a separate class for "special tolerances". This class is used when tight tolerances are needed for requirements such as serviceability, aesthetics, or assembly requirements. In the context of modular construction, special tolerances are often needed for ensuring proper assembly of modules on site (Lawson et al. 2014). However even with provision of special tolerances, there is often too much controversy over tolerance magnitudes and too few field measurements of actual construction to 
justify tolerances (Malisch 2010). For this reason, many works have explored field measured variations and reported on their tolerances (Ballast 2007). Works like these focus on reporting specific tolerances for distinct materials and applications (e.g., cast-in-place concrete joints, curtain walls on steel frames, panelling on stick-built substrates, etc.). With the exception of research conducted by Acharjee (2007) and Milberg and Tommelein (2007; 2009; 2003), there are not many research works exploring how dimensional variability accumulates in assemblies during the construction lifecycle of a project. Related research has only explored the final accumulation of dimensional variability and proposed mechanisms and tools for design, but have not conducted root cause analyses of variations with respect to required tolerance values.

\section{PROPOSED METHODOLOGY}

The proposed methodology analyzes construction processes in terms of their impact on dimensional variability of a steel framed modular assembly. Four critical impact categories are used for classifying dimensional variability, which are derived from requirements in codes and standards related to both steel and concrete construction (ACI 2002, AISC 2010a, AISC 2010b):

a) Structural safety: this is the most important class and is used to describe conditions where dimensional variability can change load paths in a structure to where materials can yield or instability arises. This category is critical for the safety of the structure.

b) Constructability (i.e., cost of manufacturing, fabrication and assembly): this class is used to ensure that the construction assembly can be economically fabricated and assembled. Trade-offs in this category are between manufacturing goals (i.e., components can be manufactured using economical processes) versus assembly and site-erection goals (i.e., modules can be easily assembled or erected into place without the use of intensive activities which would introduce rework and cause cost overruns and delays).

c) Aesthetics: this class relates to the overall perceived quality of the final constructed project. Of importance for ensuring adequate aesthetics is managing dimensional variability at joints so that the joints appear to align properly.

d) Functionality: this class is related to both the serviceability of a building, as well as the performance and alignment of subsystems.

While structural safety is the most important impact category, order of importance for remaining categories is often determined on a case specific basis. To quantify dimensional variability, 3D imaging is employed since it can yield rich and accurate dimensional information about assembly surfaces. In addition, a total station is used for cases where a laser scanner is not practical to use (refer to description in case study below). For quantifying variability of the assembly, a method called deviation analysis is employed which compares 3D as-built data with a building information model. Refer to Rausch et al. (2016) for a detailed explanations and demonstration of this method.

\section{CASE STUDY}

To demonstrate how dimensional variability can accumulate throughout a modular assembly during the construction lifecycle (i.e., from part manufacture to final erection on site) a sample case study is shown. A construction company was contracted to construct two single story 805 $\mathrm{m}^{2}$ data centers, each comprised of 16 prefabricated steel framed modules. As-built dimensional data was collected during key project stages to determine how dimensional variability accumulates and to pinpoint where tolerances are required to be more stringent to ensure 
compatibility. The key project stages considered are: (1) fabrication and assembly of structural system (floor frame with precast concrete panels, columns and roof frame), (2) transportation and handling of modules, and (3) erection. After quantifying critical dimensional variability, it is classified in terms of its impact and analyzed in terms of its contribution to overall accumulation.
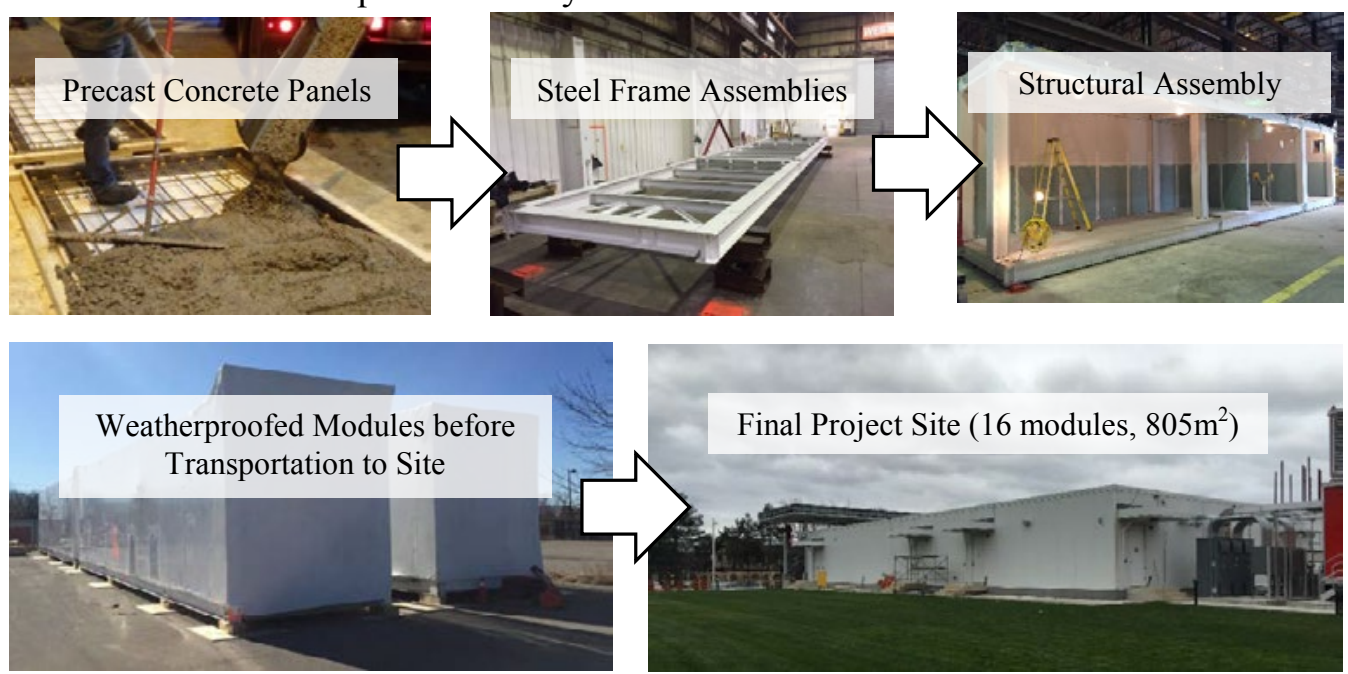

Figure 1: Case study for examining accumulation of dimensional variability.

\section{Stage 1: Fabrication and assembly of the structural system}

During fabrication of key components in the structural assembly, laser scans were taken of precast concrete panels and steel frames (note the floor is comprised of precast concrete panels placed into a steel frame). Critical variability of concrete panels was based on guidelines in the Precast/Prestressed Concrete Institute Design (PCI) Handbook: (1) overall XYZ dimensions and bowing/warping. For the steel frames, critical variability was based on guidelines in AISC Code of Standard Practice for Steel Buildings and Bridges: (1) overall XYZ dimensions for the slots and welding distortion. Using the $3 \mathrm{D}$ point cloud data, $\mathrm{XYZ}$ dimensions of panels and frame slots were calculated. These dimensions were fit with probability distribution curves and compared in order to assess the overall assembly compliance. Note that proper assembly occurs in cases where $\left(\operatorname{Dim}_{\text {panels }}<\operatorname{Dim}_{\text {frame}}\right)$. This analysis revealed that the probability of nonconformance based on intersection of the probability distribution functions is equal to $50 \%$ (Figure 2).
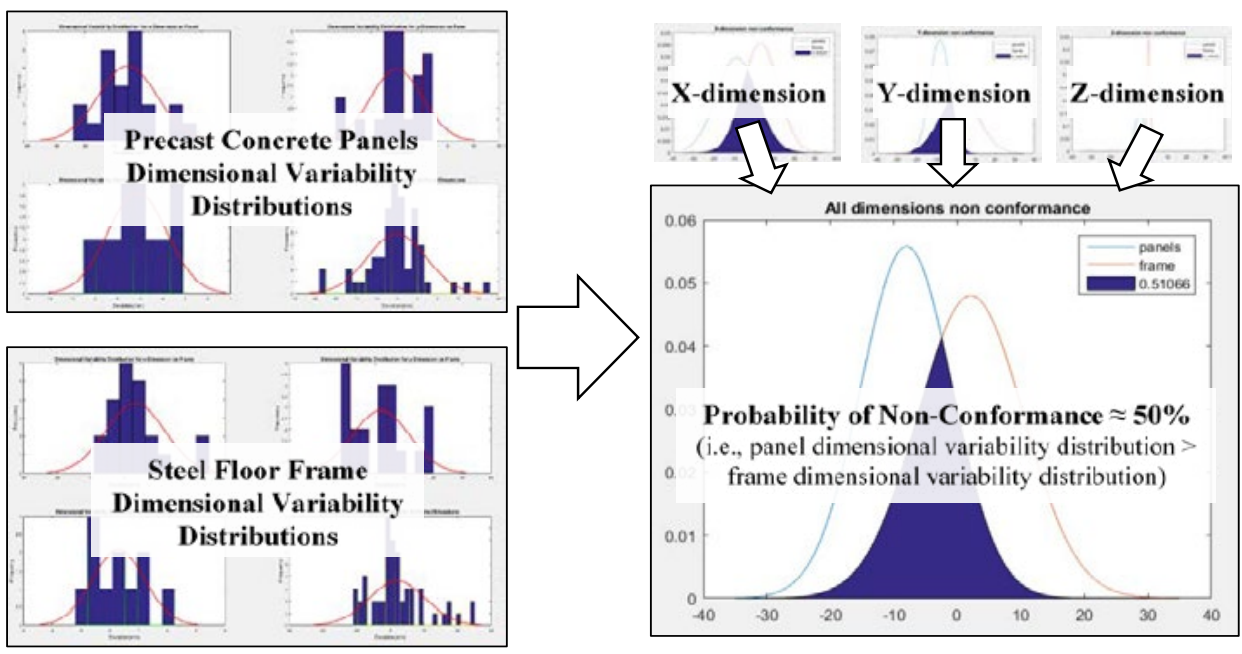

Figure 2: Assessment of assembly compliance through comparison of probability 
Dimensional variability associated with warping of concrete panels and welding distortion in the steel frames was assessed by conducting plane deviation analyses in a commercial metrology software (PolyWorks $\left.{ }^{\circledR}\right)$. The premise behind plane deviation analyses is to compute Euclidean distances from points in a 3D point cloud to a best-fit plane. Plane deviation analyses of the concrete panel surfaces (along the top, bottom and sides) revealed little warping (only $5 \mathrm{~mm}$ along the $2500 \mathrm{~mm}$ length). Examination of welding distortion in the steel frames (roof and floor) revealed slightly larger deviations -approximately $17 \mathrm{~mm}$ of warping in the roof frame, and $13 \mathrm{~mm}$ in the floor frame along the $16,000 \mathrm{~mm}$ module length (Figure 3a-b). This variability is problematic for assembling the module to adjacent modules, since connection points between modules are not in the same plane. Dimensional variability of the final structural assembly (floor frame with precast concrete panels, columns and roof frame) was quantified using a scan-vs-BIM deviation analysis (refer to Rausch et al. 2016 for explanation of this deviation analysis). This analysis (Figure 3c) revealed overall discrepancies between the as-built assembly and the asdesigned state were as large as $20 \mathrm{~mm}$.

(a) Roof Frame Distortion Pattern

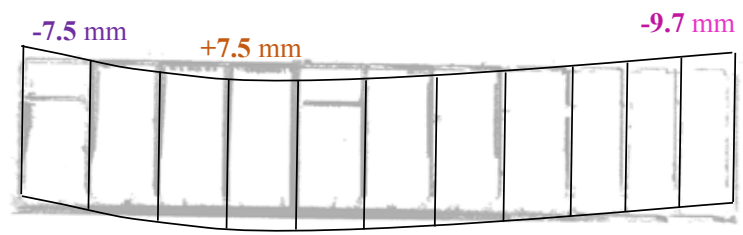

(b) Floor Frame Distortion Pattern

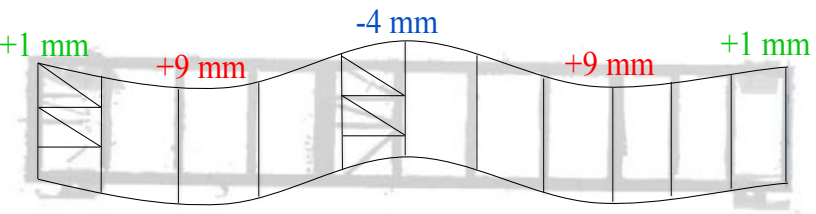

(c)

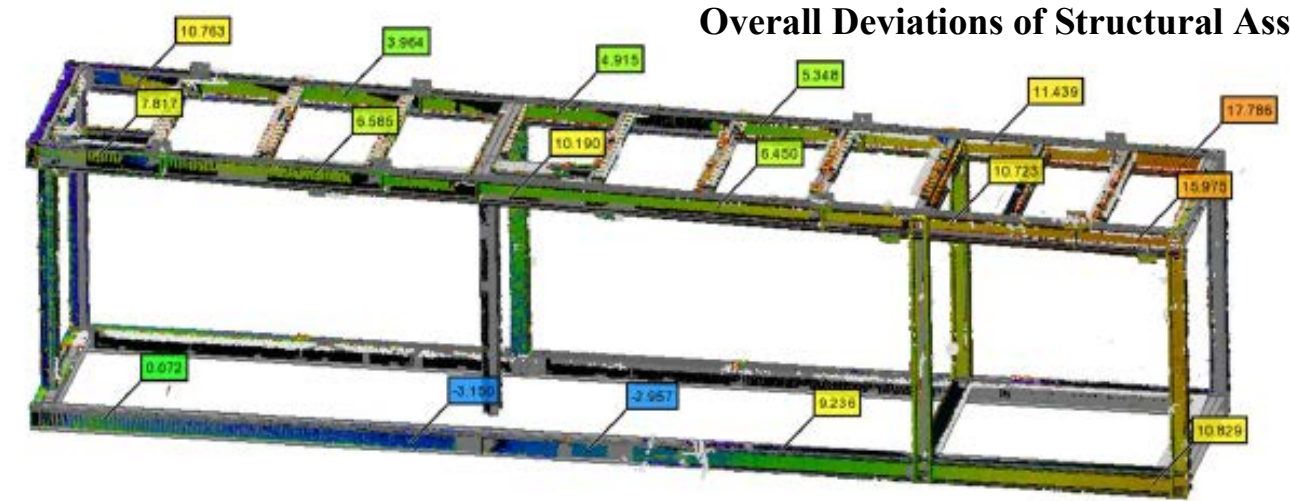

Figure 3: Quantification of dimensional variability for (a) roof frame assembly, (b) floor frame assembly, and (c) overall structural assembly. Note: (a) and (b) depict not-to-scale deviation patterns overlaid on the 3D point clouds in 'plan view'.

\section{Stage 2: Transportation and handling modules}

The second stage examines dimensional variability associated with the geometric response of modules to the following scenarios: crane handling loads, temporary support conditions and transportation loads. The geometric response of the module was assessed in terms of elastic (i.e., non-permanent) and plastic (i.e., permanent) deviations. The geometric effect of crane handling loads was quantified using a scan-to-scan deviation analysis where two 3D point clouds were collected: (1) module positioned on a framing table, and (2) module suspended by the crane. Movements of the module were controlled using supports. Deviation analysis output revealed a 3 $\mathrm{mm}$ elastic deviation, where the largest deviations occurred at the ends of the module (Figure 4a). In this project a lifting frame was used to control large plastic deviations from crane handling. The geometric effect of temporary support conditions (4 corner supports) was assessed 
to determine how much the module elastically deflects under self-weight. A plane deviation analysis revealed mid-span deflections equal to $30 \mathrm{~mm}$ (Figure $4 \mathrm{~b}$ ). While the effect of plastic deviations is obvious to the overall accumulation of dimensional variability, the effect of elastic deviations (especially as large as $30 \mathrm{~mm}$ ) influence the layout and alignment of subsystems within the module during fabrication (e.g., alignment of mechanical, electrical, plumbing systems). Finally, the geometric effect of transportation loads was quantified. Due to weatherproofing on the module, it was not feasible to use a laser scanner since the weatherproofing is flexible and therefore not an accurate representation of plastic deviations. Instead permanent targets placed on parts of the exposed structure were surveyed before and after transportation; revealing plastic deviations of $3 \mathrm{~mm}$ (Figure $4 \mathrm{c}$ ).

(a)
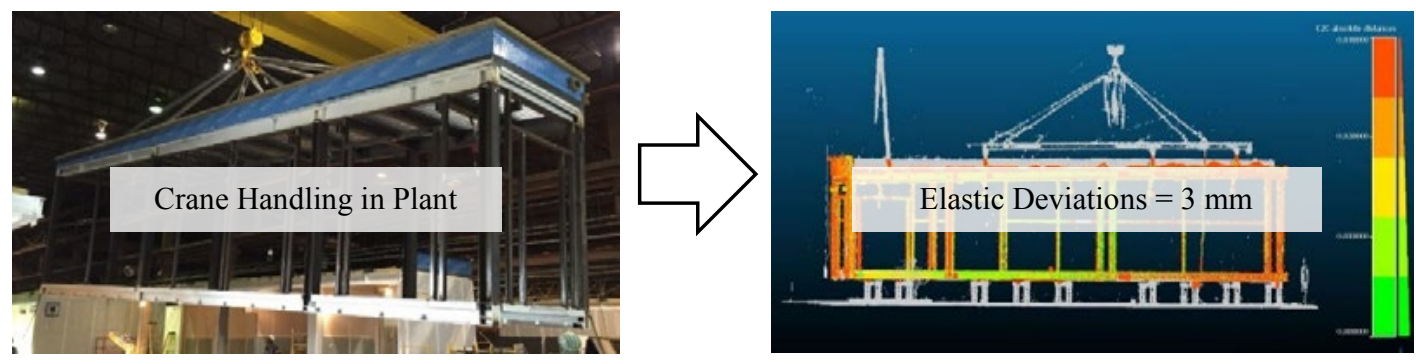

(b)
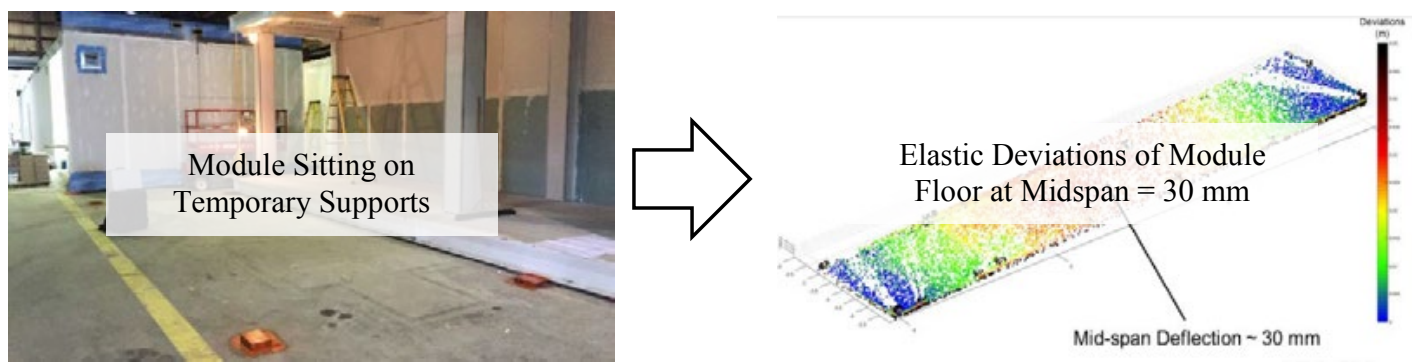

(c)
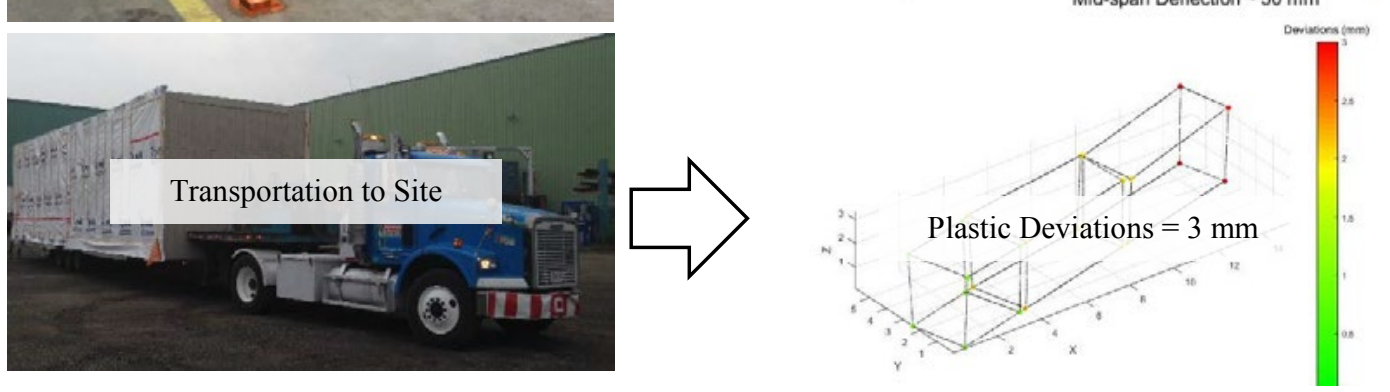

Figure 4: Quantification of dimensional variability associated with the transportation and handling of modules. (a) Elastic response to handling load. (b) Elastic response to temporary support conditions during assembly. (c) Plastic response to transportation load.

\section{Stage 3: Erection}

The final stage examines dimensional variability associated with the geometric response of the module to erection loads during fit-up and alignment on site. During erection, the spacing between modules exceeded the design specified gap (gaps between module tie-in points were as large as $50 \mathrm{~mm}$, as shown in Figure 5a). The crew installing the modules noted several cases where the final geometry of the modules did not align correctly (between modules or to the foundation). To quantify the geometric effect of erection loads, a total station was used similar to the approach taken in Stage 2. It was not feasible to use a laser scanner in this stage since there was not enough exposed structure along with walls of the module. Furthermore, use of a laser 
scanner for prolonged periods within the modules was not possible since contractors were conducting work, thus creating occlusions for the scanner. However, quantification through use of a total station revealed plastic deviations on the order of $7 \mathrm{~mm}$ due to erection loads (Figure 5c).
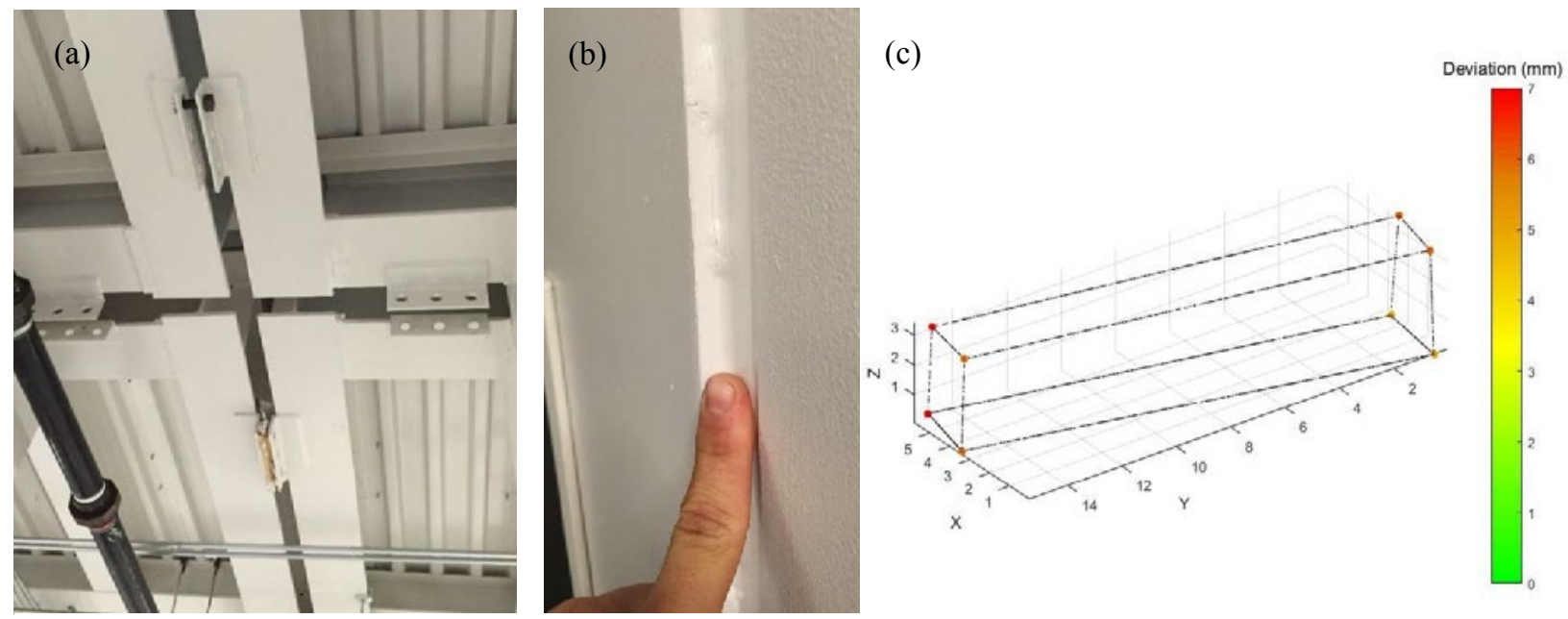

Figure 5: Quantifying dimensional variability of in-situ construction assembly: (a) large gaps between adjacent modules $(\sim 50 \mathrm{~mm})$, (b) misalignments of columns and (c) plastic deviations associated with erection loads.

\section{DISCUSSION}

The impact of dimensional variability for construction of modules in this case study can be expressed in terms of key impact categories as shown in the proposed methodology. For fabrication and assembly, the primary impact is constructability. This is evident in the nonconformance of panel and frame dimensions. To improve constructability, a trade-off between the cost for components with stricter tolerances and more precise construction processes is required. For the effect of temporary support conditions, the primary impact of dimensional variability is functionality, since large elastic deviations influence the alignment and dimensional variability of subsystems (e.g., mechanical, electrical, plumbing) and architectural components (doors, windows, etc.). The geometric response from loads due to transportation, handling and erection impact the final module geometry. These effects influence ease of assembly for modules onsite (i.e., constructability), as well as the aesthetics of the final building. Note that due to the accumulation of variability in this project, many columns were notably misaligned after construction was complete (Figure 5b), giving the perception of poor quality (i.e., aesthetics). In this project, the aspect of structural safety was not directly impacted as the result of variability.

\section{CONCLUSION}

The accumulation of dimensional variability in modular construction has distinct impacts on overall project performance in terms of structural safety, constructability, aesthetics and functionality. These categories are not exhaustive, and in some cases may include other impacts. It is important to properly identify and manage dimensional variability at key project stages in order to mitigate its impact. Trade-offs between impacts exist when specifying tolerances and 
controlling dimensional variability. The case study in this paper shows that dimensional variability can be quantified through use of a laser scanner and total station by making comparisons between as-built and as-designed data. In conclusion, design of modular construction systems needs to account for the accumulation of dimensional variability in order to holistically control its impact.

\section{REFERENCES}

Acharjee, T. (2007). "Investigating Accumulation of Tolerances and Its Impact on Reliability of Job Site Installation". MASc. University of Cincinnati.

ACI. (2002). ACI Committee 117, Commentary on Standard Specifications for Tolerances for Concrete Construction and Materials (ACI 117-06). American Concrete Institute, Farmington Hills, Michigan.

AISC. (2010a). Code of Standard Practice for Steel Buildings and Bridges (AISC 303-10). American Institute of Steel Construction, Chicago, Illinois.

AISC. (2010b). Specification for Structural Steel Buildings (ANSI/AISC 360-10). American Institute of Steel Construction, Chicago, Illinois.

Ballast, D. K. (2007). Handbook of construction tolerances. John Wiley \& Sons.

Birkeland, P. W., and Westhoff, L. J. (1971). "Dimensional Tolerances in a Tall Concrete Building." Journal Proceedings, 600-607.

Lawson, M., Ogden, R., and Goodier, C. (2014). Design in modular construction. CRC Press.

Malisch, W. R., and Suprenant, B. A. (2005). "ASCC Summary Report for the meeting of an Inter-industry Working Group on Reducing the Cost of Tolerance Compatibility Problems held February." American Society of Concrete Contractors.

W. R. Malisch. (2010). "Avoiding Tolerance Traps." http://www.cibofnyc.org/documents/2010/TolerancesMalischNY.pdf (06/20, 2016).

Milberg, C. (2007). "Tolerance Considerations in Work Structuring." Proceedings IGLC.

Milberg, C. T., and Tommelein, I. D. (2009). "Tolerance and Constructability of Soldier Piles in Slurry Walls." J.Perform.Constr.Facil., 24(2), 120-127.

Milberg, C., and Tommelein, I. (2003). "Role of tolerances and process capability data in product and process design integration." Construction Research Congress 2003, ASCE Honolulu, Hawaii, 1-8.

Milberg, C., and Tommelein, I. D. (2005). "Application of tolerance mapping in AEC systems." Construction Research Congress 2005, 1-10.

L. S. Muir. (2015). "Steelwise: on target and within tolerance." http://msc.aisc.org/globalassets/modern-steel/archives/2015/09/steelwise.pdf (06/20/16).

Posey, J. B., Dalgliesh, W. A., Eng, P., Little, A., Arch, B., Tucker, C., and ALS, P. E. (2007). "Variations in Position of Columns and Slabs." 11th Canadian Conference on Building Science and Technology.

Rausch, C., Nahangi, M., Haas, C., West, J., and Perreault, M. (2016). "Deviation Analyses: A Tool for Quantifying Dimensional Quality and Alignment in Modular Construction." 2016 Annual National Conference, Canadian Society for Civil Engineering.

Silva, P. (2012). "Movement and Tolerances." ICE Manual of Structural Design: Buildings, ICE Publishing, 267-291. 